\title{
BMJ Open Multicentre randomised controlled trial to investigate the usefulness of continuous pneumatic regulation of tracheal cuff pressure for reducing ventilator-associated pneumonia in mechanically ventilated severe trauma patients: the AGATE study protocol
}

\author{
Nicolas Marjanovic, ${ }^{1}$ Denis Frasca, ${ }^{2}$ Karim Asehnoune, ${ }^{3}$ Catherine Paugam, ${ }^{4}$ \\ Sigismond Lasocki, ${ }^{5}$ Carole Ichai, ${ }^{6}$ Jean-Yves Lefrant, ${ }^{7}$ Marc Leone, ${ }^{8}$ \\ Claire Dahyot-Fizelier, ${ }^{9}$ Julien Pottecher, ${ }^{10}$ Dominique Falcon, ${ }^{11}$ Benoit Veber, ${ }^{12}$ \\ Jean-Michel Constantin, ${ }^{13}$ Sabrina Seguin, ${ }^{14}$ Jérémy Guénézan, ${ }^{1}$ Olivier Mimoz, ${ }^{1,15}$ \\ For the AGATE study group
}

To cite: Marjanovic N, Frasca D, Asehnoune $\mathrm{K}$, et al. Multicentre randomised controlled trial to investigate the usefulness of continuous pneumatic regulation of tracheal cuff pressure for reducing ventilatorassociated pneumonia in mechanically ventilated severe trauma patients: the AGATE study protocol. BMJ Open 2017;7:e017003. doi:10.1136/ bmjopen-2017-017003

- Prepublication history for this paper is available online. To view these files, please visit the journal online (http://dx.doi. org/10.1136/bmjopen-2017017003).

Received 27 March 2017 Revised 17 May 2017 Accepted 14 June 2017

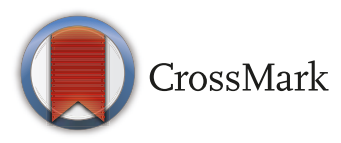

For numbered affiliations see end of article.

Correspondence to Professor Olivier Mimoz; olivier.mimoz@wanadoo.fr

\section{ABSTRACT}

Introduction Severe trauma represents the leading cause of mortality worldwide. While $80 \%$ of deaths occur within the first 24 hours after trauma, $20 \%$ occur later and are mainly due to healthcare-associated infections, including ventilator-associated pneumonia (VAP). Preventing underinflation of the tracheal cuff is recommended to reduce microaspiration, which plays a major role in the pathogenesis of VAP. Automatic devices facilitate the regulation of tracheal cuff pressure, and their implementation has the potential to reduce VAP. The objective of this work is to determine whether continuous regulation of tracheal cuff pressure using a pneumatic device reduces the incidence of VAP compared with intermittent control in severe trauma patients.

Methods and analysis This multicentre randomised controlled and open-label trial will include patients suffering from severe trauma who are admitted within the first 24 hours, who require invasive mechanical ventilation to longer than 48 hours. Their tracheal cuff pressure will be monitored either once every 8 hours (control group) or continuously using a pneumatic device (intervention group). The primary end point is the proportion of patients that develop VAP in the intensive care unit (ICU) at day 28. The secondary end points include the proportion of patients that develop VAP in the ICU, early ( $\leq 7$ days) or late ( $>7$ days) VAP, time until the first VAP diagnosis, the number of ventilator-free days and antibiotic-free days, the length of stay in the ICU, the proportion of patients with ventilator-associated events and that die during their ICU stay.

Ethics and dissemination This protocol has been approved by the ethics committee of Poitiers University Hospital, and will be carried out according to the principles of the Declaration of Helsinki and the Good

\section{Strengths and limitations of this study}

- This study will be conducted as a multicentre randomised controlled and open-label trial adequately powered to determine whether continuous regulation of tracheal cuff pressure using a pneumatic device reduces the incidence of ventilator-associated pneumonia.

- This will be the first large study on that topic with the potential of changing international recommendations on ventilation-associated pneumonia prevention.

- Study's benefits include reduced duration of mechanical ventilation and stay in the intensive care unit, improved patient prognosis and reduction in antibiotics use.

- Limitations due to the lack of masking related to the nature of the intervention are limited by a masked end point assessment.

Clinical Practice guidelines. The results of this study will be disseminated through presentation at scientific conferences and publication in peer-reviewed journals. Trial registration Clinical Trials NCT02534974

\section{INTRODUCTION}

\section{Background and rationale}

Severe trauma represents the leading cause of mortality worldwide, and is the third highest cause of death in France behind cardiovascular disease and cancer. About 46 deaths following trauma occur per 100000 inhabitants per year in France, representing 
close to $8 \%$ of all deaths. ${ }^{12}$ While $80 \%$ of deaths occur within the first 24 hours of trauma, directly related to the trauma itself, $20 \%$ of deaths occur later and are mainly due to healthcare-associated infections (HAI). ${ }^{3-6}$ More than $50 \%$ of patients that present with severe trauma will develop at least one HAI during their stay. Neurological disorders, recourse to mechanical ventilation and early post-traumatic immune suppression may explain the elevated incidence of HAI, particularly ventilator-associated pneumonia (VAP), which mainly occur in the first week following trauma. ${ }^{7-9}$

VAP represents the predominant HAI in severe trauma patients with a mean incidence of $35 \%$, which may even exceed $60 \%$ in some series, followed by catheter infections and bacteraemia. ${ }^{3-6}$ HAI account for heightened morbidity and mortality, longer stays in the intensive care unit (ICU) and increased treatment costs. In France, the additional cost is estimated at $€ 800$ million per year. ${ }^{1}$ These infections also require high use of antibiotics, and VAP alone makes up more than half of the antibiotic treatments prescribed in the ICU. ${ }^{7}$ Given the selection pressure they induce, these antibiotic therapies favour the appearance of bacterial resistance.

\section{VAP pathophysiology}

The presence of a foreign material (tracheal tube or stomach tube) facilitates colonisation of the upper airways and digestive tract by endogenous and exogenous microorganisms. Tracheal contamination originates through bacterial aspiration, resulting from the passage of secretions from around the cuff of the tracheal tube accumulating above the tracheal cuff. ${ }^{10}$ More rarely, VAP can arise from contaminated stagnant water in the ventilator circuit, use of colonised aerosols or a contaminated contiguous site (almost exclusively the pleural cavity). ${ }^{11}$

\section{Prevention measures for VAP}

Enhanced knowledge of the pathophysiology of VAP has led to the development of effective preventive strategies. The VAP prevention measures recommended by the 2008 French consensus conference on nosocomial infection prevention ${ }^{12}$ state that the orotracheal route should be used for tracheal intubation instead of the nasal route, ${ }^{7113}$ and the tracheal cuff pressure should be maintained between 25 and $30 \mathrm{~cm} \mathrm{H}_{2} \mathrm{O} .{ }^{14}{ }^{15}$ They also recommend using a sedation-analgesia algorithm that facilitates early weaning off ventilation, avoidance of unplanned extubation, ${ }^{16}$ prioritising the use of non-invasive ventilation by following the recognised indications, ${ }^{16} 17$ placing the patient in a semi-recumbent position between $30^{\circ}$ and $45^{\circ},{ }^{18} 19$ regularly decontaminating the nasal and oropharyngeal cavity through suitable mouth care ${ }^{2021}$ and starting enteral nutrition as early as possible. ${ }^{22}$ When combined with systemic antibiotic therapy, selective digestive decontamination may be recommended, but its implementation requires specification of its modalities (choice, dosing, duration of selective digestive decontamination and antibiotic therapy) and the targeted population.

Maintaining a sufficient level of pressure in the tracheal tube cuff is of critical importance. When the pressure is too low, the patient risks inhaling the accumulated subglottic secretions from the oropharynx, which is known to play a major role in VAP incidence. ${ }^{14}$ Alternatively, if the pressure is too high there is a risk of ischaemia of the tracheal mucosa and, at removal, of tracheal oedema and later tracheal stenosis. Therefore, regular monitoring of tracheal cuff pressure is recommended, but the optimal monitoring frequency has yet to be clearly determined. Underinflation and/or overinflation of the tracheal cuff often occurs in ventilated patients under intermittent manual control of tracheal cuff pressure using a manometer, which is a well-known source of morbidity and mortality. ${ }^{23}$ Recently published data favour use of automatic devices that are able to continuously regulate the tracheal cuff pressure. ${ }^{24-31}$ Even though several learned societies do not recommend their implementation for the purpose of VAP prevention in ventilated patients, recent findings in the literature tend to favour their use. ${ }^{24} 25272932$ However, these studies have several limitations, as all were monocentre studies that included a relatively small number of participants, which rarely included severe trauma patients even though they are at greatest risk for VAP, and these studies did not systematically apply other recommended VAP preventive measures, with a contestable choice for the main study end point.

Two methods of continuous regulation of tracheal cuff pressure are available: pneumatic and electronic. Published studies favour the first ${ }^{33}$ due to better reactivity with fewer periods of insufficient or excessive tracheal cuff pressure, and consequently, reduced risk of microbe inhalation and tracheal injuries, in addition to the low acquisition cost, ease of use and no electricity supply required.

\section{Objectives}

The objective of this study is to determine whether continuous regulation of tracheal cuff pressure using a mechanical pneumatic regulation device (Nosten, Leved, Paris) is superior to intermittent control of tracheal cuff pressure for reducing the incidence of VAP in severe trauma patients, who will receive all other recommended methods of VAP prevention. At an individual level, the benefits are expected to include a shorter duration of mechanical ventilation and stay in the ICU, as well as improved patient outcomes. At a collective level, in addition to reduced cost of hospital stay, the number of days on antibiotics should also decline, along with the selective pressure perpetuating the emergence of multidrug-resistant bacteria.

\section{Study design}

The AGATE trial is a multicentre randomised controlled and open-label clinical trial with two parallel groups, with the primary end point being the proportion of 


\section{Box 1 Inclusion and exclusion criteria}

\section{Inclusion criteria}

1. Patients aged at least 18 years

2. Suffering from a severe trauma as defined by an Injury Severity Score $>15$

3. With their tracheal intubated via the oral route for $<15$ hours

4. Requiring mechanical ventilation for an expected length $\geq 48$ hours.

5. Participating in a social security scheme or benefiting from such a scheme by means of a third party

Exclusion criteria

1. Patient likely to die in the 48 hours following admission

2. Having their trachea intubated via the nasal route

3. Patient intubated through a tracheal tube with subglottic secretion drainage

4. Having their trachea intubated 24 hours or more after trauma

5. Being ventilated with tracheotomy

6. Refusing to participate in the research

7. Having contraindication to the head-up position

8. Participating in another research protocol pertaining to an anti-infective treatment or to a measure decreasing risk of infection

9. Benefiting from reinforced protection or persons deprived of freedom subsequent to a legal or administrative decision, majors under legal protection

patients that develop VAP in the ICU at day 28. Randomisation will be carried out through a secure web-based randomisation system, stratified by the centre and severity of patient unconsciousness at the time of inclusion (Glasgow Coma Scale lower and higher or equal to 8).

\section{METHODS AND ANALYSIS}

\section{Participants}

Patients will be considered candidates for inclusion in the study if they meet all of the inclusion criteria and none of the exclusion criteria (box 1). Patients suffering from severe trauma admitted within the first 24 hours, who require invasive mechanical ventilation expected to last longer than 48 hours, should be included in the study. For all cases, patient inclusion will take place as soon as possible after ICU admission, during the 24 hours following trauma and/or over the 15 hours following oral intubation of the trachea. Pregnant or lactating women can be included in the study because the material used does not present any specific risk of teratogenicity.

\section{Interventions}

All of the patients included in the study will be randomised in one of the two treatment groups, which are based on the method used to monitor tracheal cuff pressure and benefit from daily monitoring throughout their hospital stay in the ICU. The following criteria will be applied to all patients and controlled throughout the duration of the study.

- Monitoring of tracheal cuff pressure of the tracheal tube either once every 8 hours in the control group or continuously in the intervention group through the use of a pneumatic device (Nosten), with the objective of maintaining pressure between 25 and $30 \mathrm{~cm} \mathrm{H}_{2} \mathrm{O}$.

- Implementation of identical sedation and weaning protocols for all centres.

- Implementation of a lung protective ventilation strategy $^{34}$ by maintaining a tidal volume of $6-8 \mathrm{~mL} / \mathrm{kg}$ of ideal body weight, positive end-expiratory pressure $>3$ $\mathrm{cm} \mathrm{H}_{2} \mathrm{O}$ and respiratory rate set to achieve normocapnia or optional moderate hypocapnia.

- According to the protocol observed in the unit, extubation should be considered as soon as: (1) the patient's Glasgow Coma Scale is >10; (2) the patient is coughing spontaneously or secondary to bronchial aspiration and (3) the level of pressure support is $<10 \mathrm{~cm} \mathrm{H}_{2} \mathrm{O}$, or spontaneous ventilation on a T-piece with $\mathrm{F}_{\mathrm{i}} \mathrm{O}_{2}$ lower than $40 \%$ is tolerated by the patient for at least $30 \mathrm{~min}$.

- No systematic changes to the respirator circuits.

- Preferential use of heat and humidity exchange filters to be changed only when soiled.

- Systematic application of bed inclination of at least $30^{\circ}$ to be monitored every 4 hours.

- Hand washing (hydroalcoholic friction) prior to any treatment and following isolation measures.

- Mouth care every 4-8 hours according to the protocol observed in the unit.

- Tracheal aspiration carried out using sterile equipment, only to be performed when required.

- Preferential oral insertion of feeding tubes.

- Starting enteral feeding as soon as possible.

- Systematic application of a glucose monitoring protocol in which the patient's blood sugar level is measured every 4 hours, the objective being to maintain the glucose level between 4 and $8 \mathrm{mmol} / \mathrm{L}$.

- Prophylactic antibiotic treatment limited to 48 hours for fractures operated within the first 6 hours.

- Curative antibiotic therapy reserved for infections for which the bacterial origin is documented, or probable, and in cases where other anti-infectious measures are insufficient, in accordance with the 2008 recommendations of the official French health authority on the proper use of antibiotics. ${ }^{35}$ An adequate dosage regimen and method of antibiotic administration is provided to ensure an optimal concentration at the site of infection. Recourse to serum assay is useful whenever possible to avoid underdosing, which is a common cause of failure, as well as to avoid overdosing, which leads to iatrogenic pathologies. Narrow-spectrum antibiotics are preferred. In cases of severe infection, antibiotic treatment should be started as soon as possible following diagnostics and microbiological sampling, and started no later than the first hour in cases of septic shock. The duration of VAP treatment should be limited to 1 week, except in cases with non-fermenting Gram-negative bacterial infections for which antibiotic therapy should continue for 2 weeks to reduce the risk of recurrence. Antibiotic associations are strictly limited to precisely defined 


\section{Box 2 American Thoracic Society definition for VAP}

VAP diagnosis is based on an association, 48 hours after the start of mechanical ventilation, of at least:

Two clinical signs among the following:

- Fever $\geq 38.0^{\circ} \mathrm{C}$ or hypothermia $\leq 36.0^{\circ} \mathrm{C}$

- Purulent endotracheal aspirations

- Hyperleucocytosis $(\geq 12000 / \mathrm{mL})$ or leucopenia $(\leq 4000 / \mathrm{mL})$

One radiological sign such as:

- A new radiographic condensation

- Modification of a previously existing radiographic condensation

And a positive bacterial analysis of the respiratory tract with cultures

of at least:

- $10^{3} \mathrm{CFU} / \mathrm{mL}$ for a brush by fibroscopy or blind protected distal sampling

- $10^{4} \mathrm{CFU} / \mathrm{mL}$ for bronchoalveolar lavage

- $10^{6} \mathrm{CFU} / \mathrm{mL}$ for tracheal aspirates

$\overline{\mathrm{CFU}}$, colony-forming unit; VAP, ventilator-associated pneumonia.

situations: the need to broaden the antibacterial spectrum; severe and microbiologically undocumented infections; Pseudomonas aeruginosa infections and bacteria and antibiotic combinations associated with a high risk of resistance emergence. These include group 3 Enterobacteriaceae (eg, Enterobacter, Serratia, Citrobacter freundii, Providencia and Morganella) and third-generation cephalosporins; Staphylococcus aureus and fluoroquinolones, rifampicin, fusidic acid or fosfomycin and Enterobacteriaceae resistant to nalidixic acid and fluoroquinolones. Antibiotic therapy should be re-evaluated between 24 and 72 hours.

- Prevention of ulcer disease by anti- $\mathrm{H}_{2}$ and proton pump inhibitors, in accordance with the protocol observed in the centre.

\section{Study outcomes}

Primary end point

- Proportion of patients that develop VAP at day 28 in the ICU according to the American Thoracic Society (ATS) definition (box 2). ${ }^{7}$

\section{Secondary end points}

- Proportion of patients that develop VAP in the ICU according to the ATS definition. ${ }^{7}$

- Proportion of patients that develop bacteraemic VAP in the ICU according to the ATS definition. Bacteraemic VAP is defined as at least one positive blood culture that is not related to any other source of infection, and at least one positive respiratory sample culture obtained within 48 hours of each other. In addition, at least one of the microorganisms isolated in the respiratory sample needs to also be isolated in the blood cultures, whereas all isolates identified in the blood cultures (except for skin contaminants) should also grow in the simultaneously obtained respiratory sample in order to meet the complete definition of bacteraemic pneumonia.
- Proportion of patients that develop early ( $\leq 7$ days) or late ( $>7$ days) VAP in the ICU according to the ATS definition. $^{7}$

- Time until the first diagnosis of VAP according to the ATS definition. ${ }^{7}$

- Proportion of patients that develop ventilator-associated events (VAE) according to the Centers for Disease Control and Prevention (CDC) definition (table 1). ${ }^{36}$

- Number of ventilator-free days.

- Number of antibiotic-free days.

- Length of stay in the ICU.

- Proportion of patients that die during their ICU stay.

- Proportion of patients that require corticosteroids or bronchodilators within 48 hours of tracheal extubation.

Attempted detection of VAP signs will be performed on a daily basis while the patient is being mechanically ventilated, in accordance with the definition established by the ATS in 2005 (box 2). ${ }^{7}$ A thoracic X-ray aimed at detecting VAP is to be carried out within a few hours whenever at least two of the following clinical signs of VAP are observed in the same clinical examination: fever $\geq 38.0^{\circ} \mathrm{C}$ or hypothermia $\leq 36.0^{\circ} \mathrm{C}$, purulent endotracheal secretions or either hyperleucocytosis $(\geq 12000 / \mathrm{mL})$ or leucopenia $(\leq 4000 / \mathrm{mL})$.

Whenever the patient meets the clinical and radiological criteria on the same day, VAP is suspected and the modified clinical pulmonary infection score will be calculated, as presented in table 2. Bacterial analysis of the respiratory tract will be carried out especially if the calculated score is $>6$. The type of sampling (bronchoalveolar lavage, blind distal sampling or tracheal aspirates) is left to the discretion of the attending physician, and depends on the procedures of the unit.

When a patient is suspected to have developed VAP, two assessors masked to the group assignment will review the case-report form and classify the case according to the accepted definition.

\section{Participant timeline}

The patients will be monitored from randomisation through to their discharge from the ICU without exceeding 60 days following inclusion. Evaluation of the assessment criteria will be carried out depending on the duration of patient stay in the ICU.

- Evaluation will be carried out on discharge from the ICU for patients with an ICU stay of $<28$ days.

- Evaluations will be carried out on day 28 and on discharge from the ICU for patients with an ICU stay of $\geq 28$ but $<60$ days.

- Evaluations will be carried out on days 28 and 60 for patients with an ICU stay of $\geq 60$ days.

Any patient who leaves the ICU will be considered to have exited the study. Any readmission into the ICU (even within 60 days following the trauma) will not be taken into account. Any event that occurs after the first 


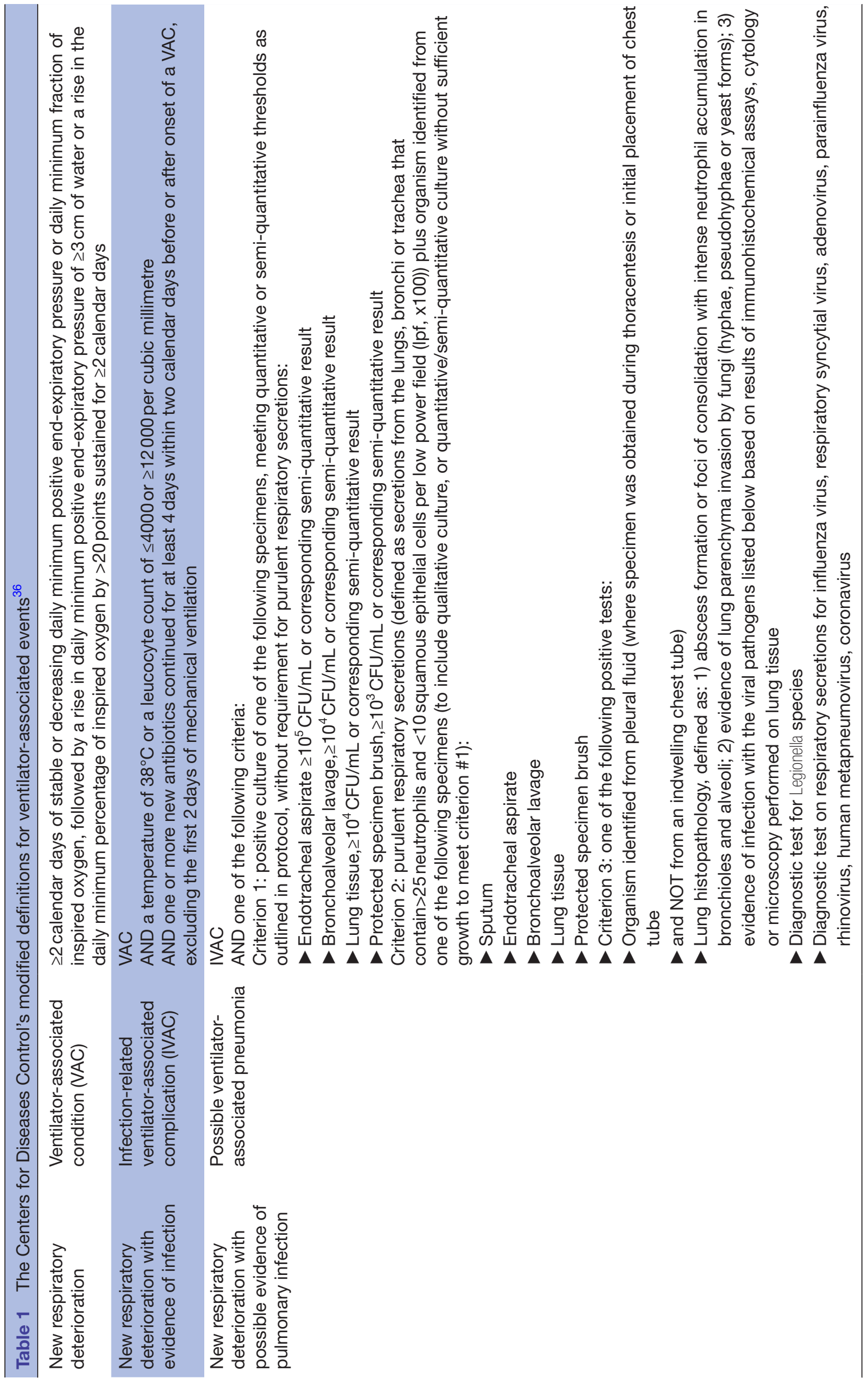


Table 2 The modified clinical pulmonary infection score for ventilator-associated pneumonia ${ }^{7}$ ARDS, acute respiratory distress syndrome.

\begin{tabular}{ll}
\hline Temperature $\left({ }^{\circ} \mathrm{C}\right)$ & \\
$\geq 36.5$ and $\leq 38.4$ & 0 \\
$\geq 38.5$ and $\leq 38.9$ & 2 \\
$\geq 39.0$ or $\leq 36.0$ & \\
Leucocytes $(\mathrm{g} / \mathrm{L})$ & 0 \\
$\geq 4$ and $\leq 11$ & 1 \\
$<4$ or $>11$ & 0 \\
\hline Tracheal secretion quantity & 1 \\
Low & 2 \\
\hline Moderate & \\
Abundant & +1 \\
\hline Tracheal secretion aspect & \\
\hline Purulent & 0 \\
\hline PaO $/$ FiO & \\
\hline$>240$ or ARDS & 2 \\
$\leq 240$ and absence of ARDS
\end{tabular}

Frontal chest radiograph

$\begin{array}{ll}\text { No infiltrate } & 0 \\ \text { Diffuse and/or heterogeneous infiltrates } & 1 \\ \text { Localised infiltrate } & 2\end{array}$

ICU discharge will not be considered in the assessment criteria.

Any patient can exit the study if he or she wishes, without having to provide justification. The quality of their care will not be altered, and the outcome will not be taken into account in the final analysis.

\section{Sample size calculation}

The number of participants ( $n=220$ in each group) is based on an estimation of VAP incidence of $20 \%$ of patients in the control group and a reduced VAP incidence (halved) in the intervention group, with a study power of $80 \%$ and $\mathrm{p}$ value of $5 \%$ in bilateral situation. The VAP incidence in the control group was chosen on the basis of an unpublished analysis of the database of AtlanRéa, a French research network devoted to studies on trauma patients. ${ }^{37} 38$

Given the recruitment potential of the centres approached to participate in the study, together with the constraints associated with the protocol, the expected inclusion duration has been set at 33 months.

\section{Recruitment}

Patients admitted into the ICUs of 15 French University Hospitals will be screened and enrolled within 24 hours of trauma. Patient enrolment will be decided by the attending physicians.

\section{Assignment of interventions and masking protocol}

A computer-generated numbered list was provided by a statistician not involved in either screening patients or the assessment of outcomes. Randomisation will be carried out using a secure web-based randomisation system with stratification by centre and severity of unconsciousness at the time of inclusion (Glasgow Coma Scale $<8$ or $\geq 8$ ), to account for differences in patient treatment between centres and the heightened VAP risk in patients with a Glasgow Coma Scale $<8$. Patients will be randomly assigned (1:1) into one of the two treatment groups, based on the method used to monitor tracheal cuff pressure. Masking of the participants and ICU staff will not be feasible due to the nature of the device, which is placed closed to the patient's face. However, the microbiologists that test the pulmonary and blood samples, the outcome assessors (NM and $\mathrm{OM}$ ) and the statisticians will be masked to the group assignment.

Data collection, confidentiality, storage and archiving of study documents

Independent clinical research assistants are available at each participating hospital to help with running the study and data collection. Study documents will be de-identified and stored for 15 years, as per the protocol for non-clinical trial notification (CTN) interventional studies. Data will be electronically stored on double password-protected computers. Hard copies of data (clinical research files) will be stored in a locked, secure office. All personnel involved in data analysis will be masked. Only the principal investigators and the statisticians will have access to the final data set.

\section{Statistical methods}

Analyses will be performed following the intention-totreat principle (all randomised patients except those that withdrew consent). Statistical analyses will take into account the stratified randomisation (centre and Glasgow Coma Scale $<8$ or $\geq 8$ ), as recommended in the 2010 Consolidated Standards of Reporting Trials (CONSORT) guidelines and other studies reported in the literature. ${ }^{39} 40$

The categorical variables will be reported as numbers and percentages, while continuous variables will be summarised using means $( \pm \mathrm{SD})$ or medians (IQR) for normally and non-normally distributed data, along with their respective $95 \%$ CIs.

Analyses of the primary end point and secondary end points related to VAP incidence will use logistic regression models adjusted for stratification factors and covariates significantly imbalanced between groups.

The cumulative event curves (time until first VAP diagnosis) will be estimated using the Kaplan-Meier procedure.

The number of ventilator-free days (VFD) is the number of days for which the patient is successfully weaned from mechanical ventilation until the end point at day 28 (or day 60). This is defined as follows: $\mathrm{VFD}=0$ for patients who died within 28 (or 60) days or required mechanical 
ventilation for 28 (or 60) or more days; VFDs=(28 (or $60)-\mathrm{x}$ ) for patients who were successfully weaned off mechanical ventilation within 28 (or 60) days, where $\mathrm{x}$ is the number of days on mechanical ventilation.

The number of antibiotic-free days (AFD) is the number of days that the living patient did not receive antibiotics over 28 (or 60) days. Any prophylactic or curative antibiotic therapy related to the trauma will not be considered.

The VFD and AFD will be assessed in the same manner, and will be compared between groups using regression models that allow adjustment for stratification factors. The other categorical secondary end points will be compared between groups using logistic regression models. Statistical analyses will all be conducted using SAS V.9.3 software.

Missing data will be described as the number and corresponding percentage for each group. The presence of any imbalance in the proportion of missing data between treatment groups will be evaluated using logistic regression models. To assess the robustness of the results in the case of missing data, sensitivity analyses will be performed with imputation of missing data (worst value such as failure, death, etc) and multiple imputation.

\section{Monitoring}

Clinical research associates will ensure that patient inclusion, data collection, registry and rapport are in line with the protocol, and that the study is conducted in accordance with the Good Clinical Practice guidelines. Furthermore, he or she will verify the following variables: patient initials, date of birth, sex, signed consent form, eligibility criteria, date of randomisation, treatment assignment, adverse events and study end points.

\section{ETHICS AND DISSEMINATION \\ Research ethics approval}

The clinical trial will be carried out in line with the principles of the Declaration of Helsinki and according to the Clinical Trials Directive 2001/20/EC of the European Parliament on the approximation of the laws, regulations and administrative provisions of the Member States relating to the implementation of Good Clinical Practices in the conduct of clinical trials on medicinal products for human use. Ethical aspects of this research project have been approved by the ethics committee of Poitiers University Hospital (France). The ethics committee of Poitiers approval cover all participant sites. The trial will be monitored by the research monitoring officer of Poitiers University Hospital.

\section{Consent}

Prior consent of the subject will not be possible; therefore, consent of the subject's legally acceptable representative will be requested. A consent form specifically designed for the subject's legally acceptable representative will be provided with documented approval or favourable opinion of the institutional review board in order to protect the rights, safety and well-being of the subject and to ensure compliance with any applicable regulatory requirements. Non-objection to participation in the study by the patient's relatives will be solicited, according to the requirements of the ethics committee.

\section{Confidentiality}

People with direct access to the data will take all necessary precautions to maintain confidentiality. All data collected during the study will be rendered anonymous. Only initials and inclusion number will be registered.

\section{Dissemination policy}

The results of the study will be released to the participating physicians, referring physicians and medical community no later than 1 year after the completion of the trial, through presentation at scientific conferences and publication in peer-reviewed journals

\section{DISCUSSION}

To the best of our knowledge, this is the first large-scale study to evaluate the usefulness of continuous pneumatic regulation of tracheal cuff pressure in a population of severe trauma patients, who will also benefit from other recommended VAP preventive measures. At an individual level, the benefits are expected to include reduced duration of mechanical ventilation and stay in the ICU, as well as improved patient prognosis. At a collective level, a lower cost of hospital stay is expected, in addition to fewer days on antibiotics, associated with a reduction in the selective pressure perpetuating multidrug-resistant bacteria.

In order to keep management practices as standardised as possible, we decided to limit the number of investigating centres to 15 university hospital centres (CHU), all of which are recognised in the field of trauma care and treat more than 50 severe trauma patients per year. These centres belong to three networks that share the common objective of promoting multicentre clinical research, consisting of AtlanRéa (Grand Ouest surgical intensive care network), AzuRéa (Grand Sud intensive care network) and TraumaBase (APHP trauma intensive care network). These 15 centres also share the same standards of care.

Previous studies that have evaluated devices aimed to continuously regulate tracheal cuff pressure have reported that these devices are efficient in controlling cuff pressure, ${ }^{41}$ and are well-tolerated by patients. ${ }^{33}$ In this setting, the choice of a pneumatic device instead of an electronic device presents several advantages ${ }^{33}$ : better reactivity with fewer periods of insufficient or excessive pressure into the tracheal cuff, and consequently, reduced risk of microinhalations and tracheal lesions; no need for an electricity supply; low acquisition cost and ease of use. Finally, the encouraging preliminary results previously reported using this device also significantly contributed to our choice. ${ }^{27}$ 
In the present study, no additional risk will be incurred by the study participants in terms of the usual therapy received during their hospital stay. Patients will be cared for as usual by their attending physicians. No supplementary examination will be imposed by the protocol. As the patient will be unable to hear the given information, information regarding the study will be delivered to his or her close relatives or friends. In accordance with the directives of the French national commission for data protection and liberties, the patient will be afforded the possibility of objecting to the use of data gathered in his or her name.

\section{Study limitations}

The incidence of VAP is the primary end point of this trial; however, VAP diagnosis may be difficult to perform in the ICU. Several criteria have been proposed, ${ }^{7642-44}$ but their reported performances are poor. ${ }^{45}$ In this trial, VAP incidence will be determined according to the 2005 ATS criteria. ${ }^{7}$ This choice could be challenged; however, these criteria have been commonly used for VAP diagnosis in the literature. We will also record VAE according to the CDC criteria, ${ }^{36}$ and compare the ATS and CDC definitions for VAP as a secondary objective. The halving of VAP rate could seems ambitious for a single intervention. However, such a finding was reported in two previous studies assessing the clinical impact of continuous control device of cuff pressure. $^{46} 47$

Because, mouth care and stress ulcer prophylaxis are based on local written protocols, they may act as potential confounding variables. However, this will be controlled by the stratification of the randomisation at the centre level and adjustment of statistical analyses in cases of differences between groups.

Finally, masking is not feasible due to the nature of the intervention. However, the end points will be assessed by two assessors masked to the group assignment in order to minimise bias.

In conclusion, this trial is the first multicentre randomised controlled open-label study adequately powered to test the hypothesis that a continuous pneumatic regulation of tracheal cuff pressure prevents VAP in mechanically ventilated severe trauma patients.

\section{Participating centres}

University Hospital of Poitiers (Professor Olivier Mimoz), University Hospital of Angers (Professor Sigismond Lasocki), University Hospital of Beaujon (Professor Catherine Paugam), University Hospital of Bicêtre (Professor Jacques Durnteau), University Hospital of Clermont-Ferrand (Professor Jean-Michel Constantin), University Hospital of Grenoble (Professor Jean-François Payen), University Hospital of Marseille (Professor Marc Leone), University Hospital of Nantes (Professor Karim Asehnoune), University Hospital of Nice (Professor Carole Ichai), University Hospital of Nîmes (Professor Jean-Yves Lefrant), University Hospital of Rouen (Professor Benoit Veber), University Hospital of Strasbourg (Professor Julien Pottecher), University Hospital of Besançon
(Professor Sébastien Pili-Floury), University Hospital of Dijon (Professor Belaid Bouhemad), University Hospital of Nancy (Professor Gérard Audibert); all in France.

\section{Trial status}

The trial is currently in progress, and the inclusion process started in July 2015. At the time of manuscript submission, 229 patients had been included. The first patient was included 2 days after registration of the study on ClinicalTrials.gov. We estimate that the last patient will be recruited in January 2018.

\section{Author affiliations}

${ }^{1}$ Department of Emergency and Prehospital Care, Centre Hospitalier Universitaire de Poitiers, Poitiers, France

${ }^{2}$ INSERM UMR1246 — Methods in Patient-Centered Outcomes and Health Research, Poitiers, France

${ }^{3}$ Centre Hospitalier Universitaire de Nantes, Anaesthesia and Intensive Care Unit, Nantes, France

${ }^{4}$ Assistance Publique des Hôpitaux de Paris, Intensive Care Unit, Hôpital Beaujon, Clichy, France

${ }^{5}$ Centre Hospitalier Universitaire d'Angers, Intensive Care Unit, Angers, France

${ }^{6}$ Centre Hospitalier Universitaire de Nice, Intensive Care Unit, Nice, France

${ }^{7}$ Division Anaesthesia Critical Care, Emergency and Pain Medicine, Centre Hospitalier Universitaire de Nimes, Nimes, France

${ }^{8}$ Assistance Publique Hopitaux de Marseille, Intensive Care Unit, Hôpital Nord, Marseille, France

${ }^{9}$ Centre Hospitalier Universitaire de Poitiers, Neuro-Intensive Care Unit, Poitiers, France

${ }^{10}$ Hopitaux Universitaires de Strasbourg, Intensive Care Unit, Strasbourg, France

${ }^{11}$ Centre Hospitalier Universitaire de Grenoble, Intensive Care Unit, Grenoble, France

${ }^{12}$ Centre Hospitalier Universitaire de Rouen, Intensive Care Unit, Rouen, France

${ }^{13}$ Centre Hospitalier Universitaire de Clermont-Ferrand, Intensive Care Unit, Clermont-Ferrand, France

${ }^{14}$ Department of Emergency and Prehospital Care, Centre Hospitalier Universitaire de Poitiers, Intensive Care Unit, Poitiers, France

${ }^{15}$ INSERM U1070, Université de Poitiers, Poitiers, France

Acknowledgements The authors would like to thank all physicians, nursing staff and clinical research associates of the participating centres for their participation in this study.

Collaborators Audrey Ambert, Gérard Audibert, Alexandre Bergis, Belaid Bouhemad, Elodie Caumon, Raphael Cinotti, Aminata Diop, Claire-Marie Drevet, Jacques Duranteau, Nathalie Dumont, Arnaud Fourcrier, Thomas Gaillard, AnneLaure Guérin, Marion Guyon, Stéphane Hecketsweiler, Nadia Imzi, Anna Lamacz, Christian Laplace, Sébastien Leduc, Anthéa Loiez, Sophie Lloret, Pauline Manhes, Jean-Pierre Payen, Sébastien Pilifloury, Antoine Roquilly, Lucie Vettoretti.

Contributors $\mathrm{OM}$ conceived the study, coordinated its design and drafted the manuscript. NM and OM wrote the manuscript. DFr, JG, KA, CP, SL, Cl, JYL, ML, CDF, $\mathrm{JP}, \mathrm{DFa}, \mathrm{BV}, \mathrm{JMC}$ and SS read and were involved in critical appraisal and revision of the manuscript. DFr provided statistical expertise. All authors approved the final manuscript prior to submission.

Funding This work was supported by a grant from the French Ministry of Social Affairs and Health under number 13-0054. Funder will not have role in the study's conception or in the data analysis.

Competing interests None declared.

Ethics approval Ethic Committee of University Hospital of Poitiers.

Provenance and peer review Not commissioned; externally peer reviewed.

Data sharing statement All data are provided in full in this paper.

Open Access This is an Open Access article distributed in accordance with the Creative Commons Attribution Non Commercial (CC BY-NC 4.0) license, which permits others to distribute, remix, adapt, build upon this work non-commercially, and license their derivative works on different terms, provided the original work is properly cited and the use is non-commercial. See: http://creativecommons.org/ licenses/by-nc/4.0/ 
(c) Article author(s) (or their employer(s) unless otherwise stated in the text of the article) 2017. All rights reserved. No commercial use is permitted unless otherwise expressly granted.

\section{REFERENCES}

1. Charvet-Protat $S$, Jarliet $A$, Préaubert N. Coût de la qualité et de la non qualité des soins dans les établissements de santé. Etat Des lieux et propositions, 2004.

2. Société française d'anesthésie et de réanimation, Société de réanimation de langue française. The risk for and approaches to control nosocomial infections in ICUs: guidelines from SFAR/SRLF task force on nosocomial infections in ICUs: guidelines from SFAR/ SRLF task force on nosocomial infections in ICUs. Reanimation 2005;14:463-71.

3. Osborn TM, Tracy JK, Dunne JR, et al. Epidemiology of sepsis in patients with traumatic injury. Crit Care Med 2004;32:2234-40.

4. Bronchard R, Albaladejo P, Brezac G, et al. Early onset pneumonia: risk factors and consequences in head trauma patients. Anesthesiology 2004;100:234-9.

5. Papia G, McLellan BA, El-Helou P, et al. Infection in hospitalized trauma patients: incidence, risk factors, and complications. J Trauma 1999;47:923-7.

6. Dahyot C, Laksiri L, Mimoz O. Pneumopathies nosocomiales. Elsevier. Conférence D'actualisation Du 47ème congrès National D'anesthésie et de Réanimation de la SFAR, 2005.

7. American Thoracic Society,Infectious Diseases Society of America. Guidelines for the management of adults with hospital-acquired, ventilator-associated, and healthcare-associated pneumonia. Am J Respir Crit Care Med 2005;171:388-416.

8. Hershman MJ, Cheadle WG, Wellhausen SR, et al. Monocyte HLADR antigen expression characterizes clinical outcome in the trauma patient. Br J Surg 1990;77:204-7.

9. Taniguchi T, Koido Y, Aiboshi J, et al. Change in the ratio of interleukin- 6 to interleukin-10 predicts a poor outcome in patients with systemic inflammatory response syndrome. Crit Care Med 1999;27:1262-4.

10. Kollef $\mathrm{MH}$. Prevention of hospital-associated pneumonia and ventilator-associated pneumonia. Crit Care Med 2004;32:1396-405.

11. Seguin $P$, Mallédant $Y$. Prévention des pneumopathies nosocomiales. Congrès national d'anesthésie et de réanimation. Les Essentiels 2008:495-506.

12. Société française d'anesthésie et de réanimation, Société de réanimation de langue française. 5 e Conférence de consensus Prévention desDes infections nosocomiales en réanimation - transmission croisée et nouveau-né exclus. Réanimation 2010;19:4-14.

13. Souweine B, Mom T, Traore O, et al. Ventilator-associated sinusitis: microbiological results of sinus aspirates in patients on antibiotics. Anesthesiology 2000;93:1255-60.

14. Feldman C, Kassel M, Cantrell J, et al. The presence and sequence of endotracheal tube colonization in patients undergoing mechanical ventilation. Eur Respir J 1999;13:546-51.

15. Subirana M, Solà I, Benito $S$. Closed tracheal suction systems versus open tracheal suction systems for mechanically ventilated adult patients. Cochrane Database Syst Rev 2007:CD004581.

16. de Lassence A, Alberti C, Azoulay E, et al. Impact of unplanned extubation and reintubation after weaning on nosocomial pneumonia risk in the intensive care unit: a prospective multicenter study. Anesthesiology 2002;97:148-56.

17. Girou E, Brun-Buisson C, Taillé S, et al. Secular trends in nosocomia infections and mortality associated with noninvasive ventilation in patients with exacerbation of COPD and pulmonary edema. JAMA 2003;290:2985-91.

18. Delaney A, Gray H, Laupland KB, et al. Kinetic bed therapy to prevent nosocomial pneumonia in mechanically ventilated patients: a systematic review and meta-analysis. Crit Care 2006;10:R70.

19. Goldhill DR, Imhoff M, McLean B, et al. Rotational bed therapy to prevent and treat respiratory complications: a review and metaanalysis. Am J Crit Care 2007:16:50-61.

20. Labeau SO, Van de Vyver K, Brusselaers N, et al. Prevention of ventilator-associated pneumonia with oral antiseptics: a systematic review and meta-analysis. Lancet Infect Dis 2011;11:845-54.

21. Tantipong $\mathrm{H}$, Morkchareonpong $\mathrm{C}$, Jaiyindee $\mathrm{S}$, et al. Randomized controlled trial and meta-analysis of oral decontamination with $2 \%$ chlorhexidine solution for the prevention of ventilator-associated pneumonia. Infect Control Hosp Epidemiol 2008;29:131-6.

22. Heyland DK, Cook DJ, Schoenfeld PS, et al. The effect of acidified enteral feeds on gastric colonization in critically ill patients: results of a multicenter randomized trial. canadian critical care Trials Group. Crit Care Med 1999;27:2399-406.

23. Valencia M, Ferrer M, Farre R, et al. Automatic control of tracheal tube cuff pressure in ventilated patients in semirecumbent position: a randomized trial. Crit Care Med 2007;35:1543-9.

24. Duguet A, D'Amico L, Biondi G, et al. Control of tracheal cuff pressure: a pilot study using a pneumatic device. Intensive Care Med 2007;33:128-32.

25. Nseir S, Duguet A, Copin MC, et al. Continuous control of endotracheal cuff pressure and tracheal wall damage: a randomized controlled animal study. Crit Care 2007;11:R109.

26. Braz JR, Navarro LH, Takata IH, et al. Endotracheal tube cuff pressure: need for precise measurement. Sao Paulo Med $J$ 1999;117:243-7.

27. Nseir S, Zerimech F, Fournier C, et al. Continuous control of tracheal cuff pressure and microaspiration of gastric contents in critically ill patients. Am J Respir Crit Care Med 2011;184:1041-7.

28. Abud TM, Braz JR, Martins RH, et al. The Lanz endotracheal tube decreases tracheal injury in dogs. Can J Anaesth 2005;52:878-82.

29. Farré R, Rotger M, Ferre M, et al. Automatic regulation of the cuff pressure in endotracheally-intubated patients. Eur Respir $J$ 2002;20:1010-3.

30. Young PJ, Burchett K, Harvey I, et al. The prevention of pulmonary aspiration with control of tracheal wall pressure using a silicone cuff. Anaesth Intensive Care 2000;28:660-5.

31. Kay J, Fisher JA. Control of endotracheal tube cuff pressure using a simple device. Anesthesiology 1987;66:253.

32. Nseir S, Lorente L, Ferrer M, et al. Continuous control of tracheal cuff pressure for VAP prevention: a collaborative meta-analysis of individual participant data. Ann Intensive Care 2015;5:43.

33. Brisson $\mathrm{H}$, Bouhemad B, Monsel A, et al. Comparaison De deux systèmes de régulation de la pression Du ballonet de la sonde d'intubation en réanimation: un système mécanique versus un système electrique. SRLF 2012.

34. Roquilly A, Cinotti R, Jaber S, et al. Implementation of an evidencebased extubation readiness bundle in 499 brain-injured patients. a before-after evaluation of a quality improvement project. Am J Respir Crit Care Med 2013;188:958-66.

35. French Health Authority. Stratégie D'antibiothérapie et prévention des résistances bactériennes en établissement de santé, 2008.

36. Robbins RA, Robbins R, Robbins R. CDC releases ventilatorassociated events criteria. Southwest Journal of Pulmonary and Critical Care 2017;14:40-1.

37. Seguin P, Laviolle B, Dahyot-Fizelier C, et al. Effect of oropharyngeal povidone-iodine preventive oral care on ventilator-associated pneumonia in severely brain-injured or cerebral hemorrhage patients: a multicenter, randomized controlled trial. Crit Care Med 2014;42:1-8.

38. Asehnoune K, Seguin P, Allary J, et al. Hydrocortisone and fludrocortisone for prevention of hospital-acquired pneumonia in patients with severe traumatic brain injury (Corti-TC): a double-blind, multicentre phase 3, randomised placebo-controlled trial. Lancet Respir Med 2014;2:706-16.

39. Moher D, Hopewell S, Schulz KF, et al. CONSORT 2010 explanation and elaboration: updated guidelines for reporting parallel group randomised trials. BMJ 2010;340:c869.

40. Kahan BC, Morris TP. Reporting and analysis of trials using stratified randomisation in leading medical journals: review and reanalysis. BMJ 2012;345:e5840.

41. Jaillette $\mathrm{E}$, Zerimech F, De Jonckheere J, et al. Efficiency of a pneumatic device in controlling cuff pressure of polyurethane-cuffed tracheal tubes: a randomized controlled study. BMC Anesthesiol 2013;13:50

42. Johanson WG, Pierce AK, Sanford JP, et al. Nosocomia respiratory infections with gram-negative bacilli. the significance of colonization of the respiratory tract. Ann Intern Med 1972;77:701-6.

43. Pugin J, Auckenthaler R, Mili N, et al. Diagnosis of ventilatorassociated pneumonia by bacteriologic analysis of bronchoscopic and nonbronchoscopic 'blind' bronchoalveolar lavage fluid. Am Rev Respir Dis 1991;143:1121-9.

44. Miller PR, Johnson JC, Karchmer T, et al. National nosocomial infection surveillance system: from benchmark to bedside in trauma patients. J Trauma 2006;60:98-103.

45. Rea-Neto A, Youssef NC, Tuche F, et al. Diagnosis of ventilatorassociated pneumonia: a systematic review of the literature. Crit Care 2008;12:R56-.

46. Lorente L, Lecuona M, Jiménez A, et al. Continuous endotracheal tube cuff pressure control system protects against ventilatorassociated pneumonia. Crit Care 2014;18:R77. 
47. Nseir S, Zerimech F, Fournier C, et al. Continuous control of tracheal cuff pressure and microaspiration of gastric contents in critically ill patients. Am J Respir Crit Care Med 2011;184:1041-7. 